stimmung mit den Ergebnissen von HABERLANDT ${ }^{3}$ ) bei Lamium purpureum und im Gegensatz zu den Befunden KandLERS $^{4}$ ) bei Bocconia cordata WILLd. [syn. Macleaya cordata (WILLD.) R. Br.] eine erhebliche irreversible Volumvergrößerung auf (Fig. 1).

Unter den von Schmucker und Fredler für Bocconia angegebenen Bedingungen konnte auch ich ebenso wie $\mathrm{KAND}-$ IER Zellteilungen nicht beobachten. Erst als submersen Kulturen einige Tage nach Versuchsbeginn, zu einem Zeitpunkt, zu dem bereits ein kräftiges Streckungswachstum der in Kultur genommenen isolierten Zellen im Gange war, eine Lösung zugesetzt wurde, die nach WHITE ${ }^{5}$ ) sowie STEWARD und SHANTz ${ }^{6}$ ) unter Verwendung von $u$. a. Cocosmilch und Dichlorphenoxyessigsäure zusammengeste it war, wurden Teilungen ausgelöst, die im Verlauf von einigen Tagen aus einzelnen Zellen kleine Zellhaufen entstehen ließen (Fig. 2). In den mit Reibsaft angesetzten Kulturen befand sich außer den Zellen noch je ein wenige Quadratmillimeter großes, einen Abschnitt des Mittelnervs enthaltendes Blattstückchen, das zuvor für 2 sec in eine Nährlösung von $60^{\circ} \mathrm{C}$ getaucht worden war.

Eine ausführlichere Darstellung der beobachteten Phänomene erfolgt an anderer Stelle. Die Versuche werden fortgesetzt.

Ich danke der Deutschen Forschungsgemeinschaft für eine Sachbeihilfe und meiner Frau für ihre wesentliche Hilfe bei der Durchführung der Versuche.

Botanisches Institut der Universität, Frankfurt a. $M$. HANS-WILLY KOHLENBACH

Eingegangen am 14. November 1958

1) Schmucker, T.: Planta 9, 339 (1929). -- ${ }^{2}$ ) Fredler, H.: Z. Bot. 33, 369 (1939). - 3) HABkrLandT, G.: Sitzungsber. Akad. Wiss. Wien, math.-naturw. Kl. 111, 69 (1902). - ${ }^{4}$ ) KanDLER, O.: Planta 38, $\left.564(1950) .{ }^{5}\right)$ WhITE, PH. R.: The cultivation of animal and plant cells. New York: The Ronald Press Company 1954. 6) STeward, F. C., E. M. Shantz: In Wain, R., L., and F. WightMAN: The chemistry and mode of action of plant growth substances. London: Butterworths Scientific Publications 1956.

\section{Zur Persistenz der Chromosomenspindelfaser}

$1947^{1 \text { a) }}$ hat der eine von uns die Persisten $z$ der Chromosomenspindelfaser in der Interphase postuliert und $1957^{1 \mathrm{~b}}$ ) erneut auf diese Vorstellung hingewiesen. Unabhängig hiervon wurde 1957 von den beiden anderen Autoren ${ }^{2}$ ) eine gleichartige Arbeitshypothese aufgestellt; die hierfür sprechenden Argumente sind inzwischen ausführlich dargelegt worden ${ }^{2 b}$ ). Aus zahlreichen Diskussionen mit Kollegen ergaben sich Fragen und Einwände, die sich vorwiegend auf das Gebiet der Meiose bezogen, die in den bisherigen Arbeiten von $H$. und $R$. LexTRt ${ }^{2 a, b}$ ) nicht behandelt wurde. Im Gegensatz hierzu waren jedoch bei REsENDE ${ }^{1 \mathrm{~b}}$ ) die Vorgänge der Meiose ausschlaggebend für die Entwicklung der Vorstellung der Persistenz der Chromosomenspindelfaser. Die Meiose fügt sich nicht nur zwanglos dieser Hypothese ein, sondern die geordnete Auseinanderführung der Schwesterchromatiden von der Anaphase I bis zur Anaphase II erscheint uns nur mit dieser Vorstellung deutbar, wenn zu diesem Zeitpunkt bereits die völlige Trennung nicht nur der Arme, sondern auch der Kinetochoren der Schwesterchromatiden vorliegt, wie es bei verschiedenen Pflanzenarten cytologisch eindeutig nachgewiesen wurde $\left.\left.{ }^{1 a}, c, d,{ }^{3}\right),{ }^{4}\right)$.

Ein Einwand gegen die Möglichkeit einer Persistenz der Chromosomenspindelfaser scheint sich aus einigen Beobachtungen an Chromosomen mit diffusem Kinetochor und dem Phänomen der Umorientierung von Univalenten in der Meiose zu ergeben. Die vorgeschlagene Deutung einer Auflösung und Neubildung der Chromosomenspindelfasern. bietet zwar eine formale, jedoch keine kausale Interpretation dieses Vorganges an. Die von ÖSTERGREN ${ }^{1 c}$ ) beobachtete Umordnung der Chromosomen von Luzula von einer gekreuzten Lagerung in Prometaphase $z \mathfrak{n}$ einer paralielen Anordnung in Metaphase läßBt sich auch ohne eire Auflösung und. Neubildung der Fibrillen durch einen verschiedenen Spannungszustand der Fibrillen deuten ${ }^{2}$ ). Die von Noronha-WAGNER und CASTRO $\left.{ }^{6}\right)$ beobachtete ungewöhnliche Orientierung der Chromosomen von Luzula während der Meiose wurde abweichend von der Interpretation dieser Autoren von RESENDE ${ }^{1}$ ) als nux durch Agglutination bedingt erkannt.

Auch die sog. Umorientierung der Univalente ${ }^{7}$ ), ${ }^{8}$ ) scheint uns mit der Persistenz der Chromosomenspindelfaser vereinbar zu sein. Die Problemstellung liegt hier in der Frage, wie es zu der anfänglichen Fehlorientierung kommt, d.h. der vorïbergehenden Verlagerung des Univalents zum falschen Pol. Diese könnte etwa durch eine passive Verschleppung (Anlagerung an andere Chromosomen) oder durch die Bildung einer nicht permanenten Faserverbindung zwischen dem Chromosom und dem, falschen" Pol bewirkt sein. Diese zeitweilige Faserverbindung wirde sich jedoch grundsätzlich von der permanenten Faser unterscheiden, die dafür entscheidend ist, welchem Pol das Univalent endgültig zugeordnet wird.

Eine Neubildung von Chromosomenspindelfasern würde weiter notwendig anzunehmen sein, wenn die Eizelle nicht über ein Centriol verfügen würde und die mütterlichen Chromosomen sich an Spindelfasern heften müßten, die vom Centriol des Spermas gebildet wären. Bereits FOI ${ }^{9}$ ). HERT$\left.W_{I G}{ }^{10}\right)$ u. a. [s. $\left.\left.{ }^{10}\right)\right]$ haben Centriolen in Eizellen nachgewiesen und Fol hat die Bewegungen der kombinierten väterlichen und mütterlichen Centriolen als , le quadrille des centres" beschrieben. Man wäre gezwungen, bei der Parthenogenese eine de-novo-Bildung einer so komplizierten Organelle wie des Centriols anzunehmen, wenn das Centriol in der Fizelle fehlen würde. Die Schwierigkeit oder sogar Unmöglichkeit des färberischen Nachweises der Centriolen in der Eizelle bestimmter Objekte schließt deren Vorhandensein nicht aus [bei Pflanzenzellen s. Koopmans ${ }^{11}$ )]. Es sei daran erinnert, da $\$$ man in den Fäden von Anteridiophoren (Characeae) ${ }^{12}$ ) und in Pollenschläuchen ${ }^{13}$ ) nur aus der Orientierung der sichtbaren Chromosomen auf die Anwesenheit des nicht darstellbaren Spindelsystems schließt.

Botanisches Institut der Universitä, Lissabon

Fí́vio Resende

Institut für Experimentelle Krebstorschung der Universität, Heidelberg

Hans LetTré und Renate LetrRé

Eingegangen am 29. November 1958

1) Resende, F.: Portug. Acta Biol. A a) 2, 1 (1947); b) 5, 75 (1957). c) Bol. Soc. Portug. Ci. Nat. 4, (2a Sér.) 252 (1953). d) Rev. Biol. 1, 93 (1956). - 2) LeTTRE, H., u. R. LetTRE: a) Naturwiss. 44 406 (1957). - b) Rev. Hémat. 13, 337 (1958). - c) Veröff. in Vorb. - 3) Resende, F., u. M. Manarte: Portug. Acta Biol. A 3, 219 (1951). - ${ }^{4}$ ) LiMA-DE-FARIA, A.: Heteditas 42, 85 (1956). 5) OSTERGReN, G.: Hereditas 35, 445 (1949). - 6) NoronEA-WAG-

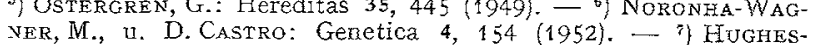
Schrader, S.: Biol. Bull. 85, 265 (1943). - - D) Dietz, R.: Chromosoma 9, 359 (1958). - 9) FoL, H.: Arch. Sci. physiques natur. 25, 214 (1891). - 10) HerTwig, R. Verh, dtsch, zool. Ges. 1892, 107. i1) Koophaxis, A.: Naturwiss. 45, 66 (1958). - 12) Mendes, E. Portug. Acta Biol. A 1, 251 (1946). 13) Raghavan, T.S., K. R. Venkatasubbaan u. H.V. WulfF: Cytologia [Japan] 9, 389 (1939).

\section{Gibberellin Modifications of Photoperiod Controlled Growth} in Herbaceous Plants

During the course of experiments ${ }^{1}$ in which numerous plant species were treated with gibberellin, growth responses were not always reproducible. Marked differences in behavior occurred in greenhouse-grown plants treated during the fall, winter and spring. Similariy the results from winter green house experiments were seldom duplicable under field conditions in the summer. These observations suggested that photoperiod was an important factor controlling plant response to gibberellin; and that the greatest percentage increases in vegetative extension and gains in weight of gibberellin treated plants occurred following exposure to a short photoperiod. Accordingly, representative groups of plants of several species were treated with gibberellin and exposed daily to both the prevailing (9-11 hour) winter photoperiod, and to 18 hours of light in which the normal photoperiod was artificially extended by incandescent lighting providing 10 to 50 foot candles at the leaf surfaces.

Results from several greenhouse experiments are summarized in Table 1. The data for heights reveal; a) that when plants were exposed to short photoperiods an effect on vegetative extension equivalent to, or greater than that from, exposure to a long photoperiod was induced with gibberellin, and, $b$ ) the most pronounced increases in height occurred when gibberellin was applied to plants exposed to short days, although significant increases usually occurred under long days. As an exception to the latter, tall peas showed little if any height response to gibberellin if grown under long days, but a marked effect at a short photoperiod. A partial or complete replacement of the effects of long days by gibberellin also occurred in dry matter accumulation for plants subjected to a 9 to 11 hour photoperiod. Whereas some 